\title{
ПРИМЕНЕНИЕ МЕТОДА ФДМР ДЛЯ ИЗУЧЕНИЯ ХЛОРОФИЛЛОПОДОБНЫХ МОЛЕКУЛ
}

1. Магнитные свойства нижнего возбужденного триплетного состояния в последние годы успешно изучаются методом оптически детектированного микроволнового резонанса (ОДМР) в нулевом поле. Хлорофиллоподобные молекулы исследовались этим методом по регистрации флуоресценции или $T-T$-поглощения $\left[{ }^{1}\right]$. Детектирование микроволнового резонанса непосредственно по изменению интенсивности фосфоресценции у данного класса соединений затруднено из-за малого выхода фосфоресценции и расположения ее в далекой красной области. Применив светосильный монохроматор и фосфороскопическую регистрацию сигнала, нам удалось получить спектры ОДМР для хлорофилла (Хл) и близких ему соединений $\left[{ }^{2}\right]$. Эти измерения дали дополнительную информацию об относительных излучательных способностях подуровней триплета. Например, для Хл $a$-уровень $(z-$ внеплоскостная ось) заметного вклада в излучение не дает, а излучательная способность $x$-уровня заметно больше, чем $y$-уровня.

Преимущество метода ОДМР перед известной техникой ЭПР состоит в сочетании магнитных резонансов с оптической селективностью детектируемых молекул. Как известно, спектры фосфоресценции хлорофиллоподобных молекул бесструктурны, а в спектрах флуоресценции обнаруживаются квазилинии из-за устранения неоднородного уширения при селективном лазерном возбуждении. В [ $\left.{ }^{3}\right]$ рассматривается зависимость частоты микроволнового резонанса от длины волны регистрации флуоресценции при селективном возбуждении хлорофиллоподобных молекул.

2. В настоящем сообщении мы обращаем внимание на методику флуоресцентного детектирования микроволнового резонанса (ФДМР) и рассматриваем возможности ФДМР-спектрометра на примере некоторых хлорофиллоподобных молекул. Известно, что вызванные поглощением СВЧ-излучения изменения интенсивности флуоресценции лежат в пределах $0,1-1 \%$. Для достижения достаточного отношения сигнал/шум необходимо усреднение по большому числу циклов измерения. С целью уменьшения общего времени накопления сигнала выгодно регистрировать сильные фототоки. Обычно это делается многократным усреднением аналогового фотосигнала $\left.{ }^{1}{ }^{1}\right]$. Мы предпочли метод счета фотонов, освобождаясь тем самым от интегрирующего влияния $R C$-фильтров при измерении кинетики микроволнового резонанса. Благодаря применению быстродействующей электроники (с полосой пропускания до 100 Мгц) нелинейность счета фотонов не превышала $1 \%$ при фотосигнале $10^{6}$ uмn $/ \mathrm{c}$ и $10 \%$ при $10^{7}$ uмn $/ \mathrm{c}$.

Принимались меры для уменьшения уровня шумов от всевозможных источников. Лазерная накачка флуоресценции стабилизировалась по интенсивности генерации света. Для устранения флуктуаций от пузырьков кипения жидкого гелия в оптическом криостате эксперименты проводились при температуре ниже $\lambda$-точки или в парах жидкого гелия 


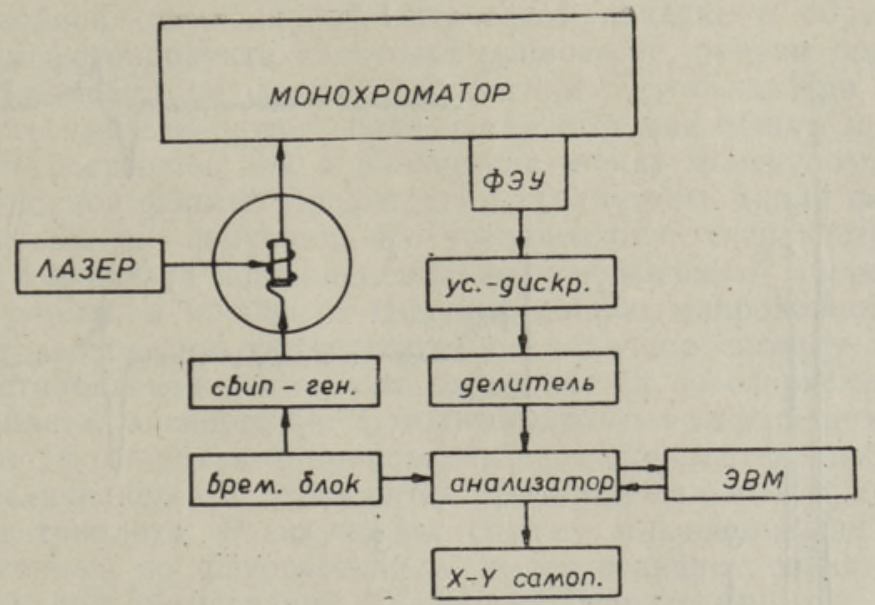

Рис. 1. Блок-схема ФДМР-спектрометра (сокращения приведены в тексте).

при 5 К. Темновой ток ФЭУ уменьшался охлаждением парами жидкого азота.

ФДМР-спектрометр (рис. 1) построен на базе оптического спектрометра, к которому прибавляется источник СВЧ-поля. Оптическая селективность получается с помощью источников лазерного возбуждения непрерывного действия и избирательной регистрации флуоресценции через монохроматор ДФС-24. Выходящее из монохроматора излучение детектируется фотоумножителем ЕMI 9816B, дающим одноэлектронный фотоимпульс длительностью не более 10 нс. Быстродействующая система регистрации сигнала состоит из усилителя-дискриминатора в счетчике фотонов 5C1 (Brookdeal), стократного делителя частоты на базе частотомера ЧЗ-54 и многоканального анализатора импульсов LP 4840 (Nokia) в режиме многократного накопления сигнала.

Источником СВЧ-поля является свип-генератор НР 8620С/86222A (Hewlett-Packard), выходная мощность которого достигает $40 \mathrm{mBT}$. Держатель объекта в криостате заменен коаксиальной линией, нагрузкой которой служит объект в спиральной замедляющей системе. Временной блок синхронизирует свип микроволнового генератора с разверткой многоканального анализатора в режиме усреднения. ФДМР-спектрометр снабжен двухсторонним каналом обмена информации между анализатором и ЭВМ ЕС 1010, что позволяет разлагать кинетические кривые резонанса на многоэкспоненциальную зависимость в ходе эксперимента. Установка может работать без присмотра в течение нескольких часов.

3. Нижнее возбужденное триплетное состояние порфиринов изучалось методом магнитных резонансов начиная с пионерских работ 3 . П. Грибовой, Л. П. Каюшиным [ $\left.{ }^{4}\right]$ и другими $\left[{ }^{5}\right]$. Нами исследовались методом ФДМР мезопорфирин-IX, копропорфирин-I и протопорфирин-IX (ППФ) в замороженных растворах н-октана. На рис. 2 слева приведен 0,0 -мультиплет Шпольского, полученный при неселективном лазерном возбуждении (4965 А) для ППФ. Справа показаны спектры ФДМР, зарегистрированные на разных линиях в мультиплете. В спектрах ФДМР наблюдаются два близко расположенных резонанса в области 1,4 Гәц, причем при переходе с одной линии флуоресценции на другую в пределах всего мультиплета изменяется знак одного резонанса. Более того, низкочастотный резонанс в спектре 3 становится высокочастотным в 

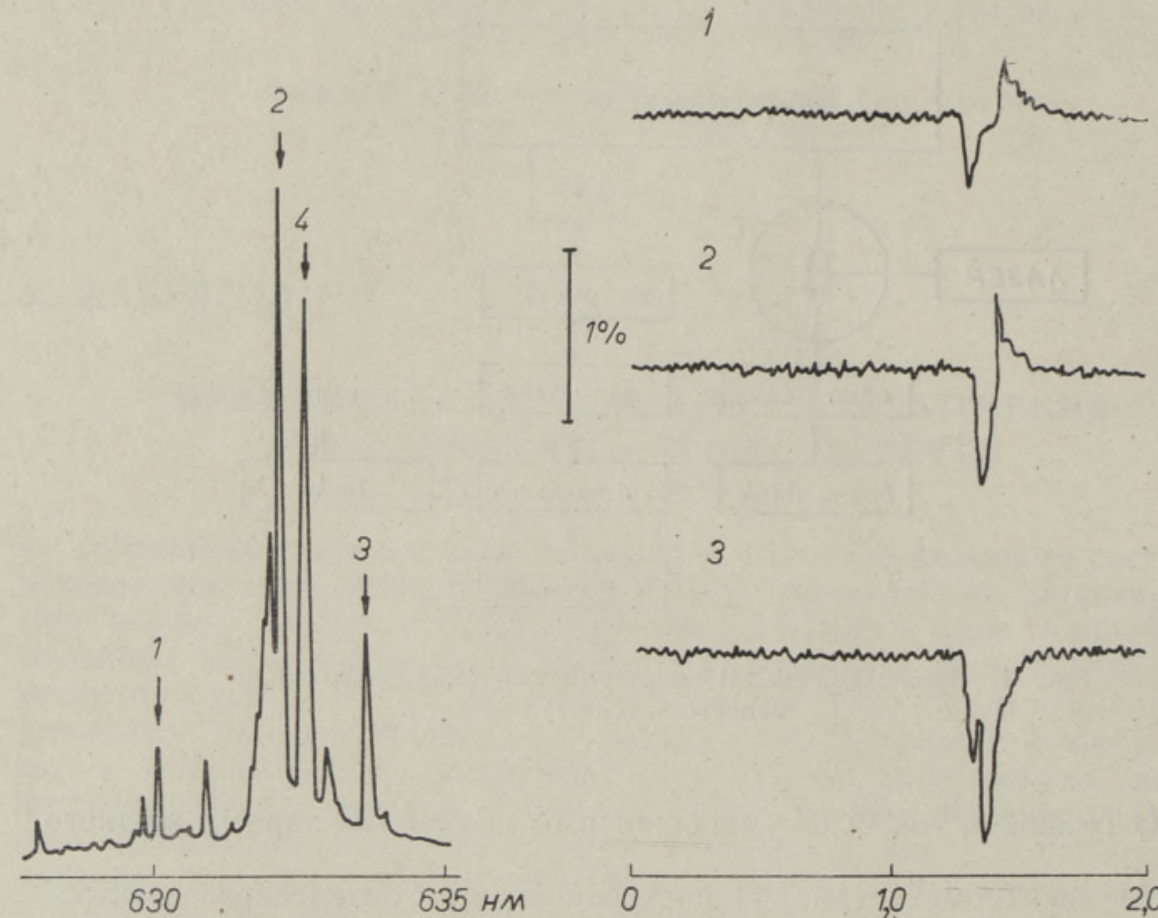

3

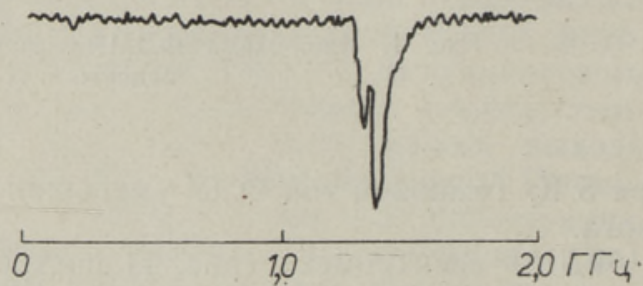

Рис. 2. (0,0)-Мультиплет Шпольского (слева) в спектре флуоресценции и спектры. ФДМР (справа) для протопорфирина-IX в н-октане.

спектре 2, и наоборот. В спектре 1 расстояние между резонансами увеличено. Объяснение такого поведения резонансов основывается на измерении кинетики для каждого из них. Двухэкспоненциальная аппроксимация пары кинетических кривых дала времена жизни подуровней триплета для ППФ $\tau_{x}=4$ мc, $\tau_{y}=15 \mu c, \tau_{z}=150$ мс при регистрации сигнала флуоресценции на линии 2 ( $x, y$ - плоскостные оси). При переходе на другие линии флуоресценции $\tau_{z}$ относительно постоянно, а $\tau_{x}$ и $\tau_{y}$ изменяются в пределах $50 \%$.

Используя величины нуль-полевого расщепления $D$ и $E$, мы наблюдали переходы $D-E$ и $D+E$, причем оказалось, что величина $|D|=$ $=1,38$ Ггц относительно мало меняется при переходе от одной компоненты Шпольского к другой, а величина $E$ варьирует в пределах $0- \pm 0,07$ Гәци и меняет знак. Магнитная неэквивалентность компонент Шпольского говорит в пользу того, что они принадлежат примесным центрам, где молекула по-разному ориентирована в кристаллической матрице н-октана. Надо отметить, что при регистрации флуоресценции на линии 4 микроволновых резонансов вообе не наблюдалось. Вероятно, для соответствующих центров величина $E \approx 0$, а заселенности подуровней таковы, что резонансы с почти равными частотами компенсируют друг друга.

Аналогичные результаты были получены для мезопорфирина и копропорфирина, у которых, однако, мультиплеты линий флуоресценции еще сложнее.

4. При изучении выжигания провала на коротковолновом крае спектра флуоресценции феофитина $a$ (Фф $a)$ обнаруживался слабый максимум при 650 нм, который был приписан фотопродукту Фф $a\left[{ }^{6}\right]$. Нас заинтересовала роль триплетного состояния при его образовании. 
При двойной стационарной оптической накачке в образовании и разложении фотопродукта наступает равновесие, причем определенное количество молекул находится в триплетном состоянии. При наложении резонансного микроволнового поля изменяется как общая заселенность триплетного состояния, так и равновесие между молекулами фотопродукта и основной формы. Предполагая замкнутость цикла фотохимического превращения, получаем, что увеличение интенсивности флуоресценции фотопродукта пропорционально уменьшению числа молекул основной формы, и наоборот. Индуцированное микроволновым полем нарушение равновесия соответствует по величине сигналу ФДМР, но время восстановления равновесия определяется не скоростью дезактивации триплета, а скоростью фотохимического превращения. Если же образование фотопродукта происходит через триплетное состояние, то эффект увеличивается - становится пропорциональным изменению заселенности триплета. В последнем случае микроволновой резонанс, детектированный по флуоресценции, имеет величину сигнала порядка получаемой при регистрации ОДМР непосредственно по фосфоресценции.

Для Фф $a$ в эфире при одновременном возбуждении линиями 6471 и $6764 \AA \mathrm{Kr}^{+}$-лазера и регистрации флуоресценции на длине волны $650 \mathrm{mM}$ нарушения фотохимического равновесия, индуцированного микроволновым полем, не наблюдалось. Отсутствие заметного эффекта говорит в пользу того, что образование фотопродукта происходит не через триплетное состояние. Наблюдению эффекта, аналогичного ФДМР, препятствовала низкочастотная флуктуация флуоресценции при двойном оптическом возбуждении и спектрально-селективной регистрации $(\Delta \lambda=$ $=5 \AA)$. Удалось зарегистрировать спектры ФДМР для Фф $a$ и его фотопродукта в эфире при возбуждении люминесценции только одной линией $6471 \AA$, когда существовало стационарное количество фотопродукта. На длине волны флуоресценции 670 нм наблюдался один резонанс при $0,96 Г г ц$, а на 650 нм - два резонанса при 0,90 и 1,10 Ггц. На основе изменения спектров ФДМР можно заключить, что ответственные за флуоресценцию 650 и 670 нм примесные центры различаются не только влиянием матрицы растворителя (неоднородное уширение спектра люминесценции), но и химическим строением примесного центра молекулы Фф $a$, что подтверждает вывод $\left[{ }^{6}\right]$ o ее фотохимическом превращении.

Автор благодарен Р. А. Авармаа за постоянное внимание к работе и К. Х. Маурингу за участие в экспериментах.

\section{ЛИТЕРАТУРА}

1. Clarke, R. H., Connors. R. E., Schaafsma, T. J., Kleibeuker, J. F., Pla te n k a m p, R. J., J. Amer. Chem. Soc., 98, № 12, 3674-3677 (1976).

2. А в а р м а а Р. А., С у й са лу А. П., Изв. АН СССР, сер. физ., 44, № 4, 843-847 (1979).

3. Avarmaa, R., Scha a f $\mathrm{ma}$ a, T. J., Chem. Phys. Lett., 71, № 2, 339-344 (1980).

4. Гр и бов в 3. П., К а юш и н Л. П., Успехи химин, 41, № 2, 287-320 (1972).

5. Van D or p, W. G., S ch a f $\mathrm{s} m$ a, T. J., S o m a, M., van der W a a ls, J. H., Chem. Phys. Lett., 21, № 2, $221-225$ (1973).

6. M a u ring, K., A va rma a, R., Chem. Phys. Lett., 81, № 3, 446-449 (1981),

Институт физики

Академии наук Эстонской ССР 


\section{FDMR-MEETTODI RAKËNDAMINE KLOROFULLISARNASTE MOLEKULIDE UURIMISEKS}

On kirjeldatud fluorestsentsi järgi detekteeritud mikrolaine resonantsi (FDMR) katseseadet, milles kasutatakse spektraalselt selektiivset laserergastust ja footoniloendust, ning toodud mõõtmistulemused $n$-oktaanis lahustatud protoporfüriin-IX kohta. Viimase resonantssagedused ja FDMR-signaali märgid osutusid sōltuvaiks fluorestsentsi lainepikkusest. On vaade!dud FDMR-meetodi sobivust feofütiini $a$ molekulide fotomuundumise jälgimiseks.

\section{A. P. SUISALU}

\section{APPLICATION OF FDMR TECHNIQUE FOR STUDYING CHLOROPHYLL-LIKE MOLECULES}

An experimental set-up of the fluorescence detection of microwave resonance (FDMR) by a site-selective laser excitation and photon-counting system is described. The results of the measurements of protoporphyrin-IX in n-octane are presented, where the resonance frequencies as well as the signs of the FDMR signal depend on the fluorescence wavelength. A possibility of using the FDMR method for detecting the phototransformation of pheophytin $a$ molecules is considered. 